\title{
The association between chronic undernutrition and malaria among Ethiopian children aged 6 - 59 months: A facility-based case-control study
}

\author{
H Y Hassen, ${ }^{1}$ BSc, MPH; J H Ali, ${ }^{2} \mathrm{MD}, \mathrm{MSc}, \mathrm{CRM}, \mathrm{CME}$ \\ ${ }^{1}$ Department of Public Health, College of Health Sciences, Mizan Tepi University, Mizan Teferi, Ethiopia \\ ${ }^{2}$ School of Public Health, College of Health Sciences, Addis Ababa University, Ethiopia \\ Corresponding author: $H$ Y Hassen (abdulhamidy71@gmail.com)
}

\begin{abstract}
Background. Malaria and undernutrition remain two major causes of childhood mortality in sub-Saharan Africa, including Ethiopia. The synergetic relationship between undernutrition and infection is widely documented but the relationship with malaria remains controversial. More studies are needed to address this and provide information to develop effective strategies for malaria control and prevention of undernutrition. Objective. To assess the relationship between malaria and chronic undernutrition in children aged 6 - 59 months at Bahir-Dar special zone, Ethiopia.

Methods. A facility-based case-control study was employed to assess the relationship between malaria and chronic undernutrition at Bahir-Dar. A total of 621 children aged between 6 and 59 months, with a 1:3 ratio of confirmed malaria cases to controls, were enrolled. Clinical data and anthropometric measurements were taken, and blood film taken and examined. Anthropometric data were converted into nutritional indices using World Health Organization Anthro software version 3.2.2 and exported to SPSS for cleaning and analysis. Results. Prevalence of stunting and underweight was 50.3\% and $34.2 \%$ among cases, respectively. Stunting and underweight were significantly associated with confirmed cases of malaria after sociodemographic and other variables were controlled. Other important predictors were rural residence, sleeping under long-lasting impregnated nets, and using indoor residual spray.

Conclusion. Chronic undernutrition was closely associated with malaria infection. Major predictors for contracting malaria were stunting, underweight, rural residence, not using long-lasting impregnated nets and indoor residual spray, male sex and low educational status of mother/caregiver. Integrated actions targeting these factors are necessary to reduce the prevailing problem.
\end{abstract}

S Afr J Child Health 2016;10(1):63-67. DOI:10.7196/SAJCH.2016.v10i1.1052

Malaria is the most significant human parasitic disease and remains a major cause of morbidity and mortality worldwide, particularly in developing countries. It affects children below the age of 5 years and pregnant women more severely. Globally, an estimated 3.3 billion people were at risk of malaria, with $\sim 80 \%$ of cases and $90 \%$ of deaths in 2011 occurring in the World Health Organization (WHO) African region. ${ }^{[1]}$

Hunger and malnutrition also remain among the most devastating problems facing the majority of the world's poor and needy, and continue to dominate the health of the world's poorest nations. The United Nations Food and Agriculture Organization estimates that nearly 870 million people, or one in eight people globally, suffered from chronic undernourishment between 2010 and 2012. ${ }^{[2]}$ Nearly $30 \%$ of the population of the developing world, including infants, children, adolescents, adults and older persons, are currently suffering from one or more of the multiple forms of malnutrition. ${ }^{[3]}$ Based on the 2013 United Nations Children's Fund report, 38\% of children below the age of 5 years suffer from chronic malnutrition or stunting in sub-Saharan Africa, with malaria and undernutrition being the two major causes of childhood mortality. ${ }^{[3]}$

In Ethiopia, both undernutrition and malaria are major public health problems, with an estimate of 55.7 million people (68\% of the population) at risk for malaria, and nearly $80 \%$ of the existing 736 districts malaria endemic. ${ }^{[4]}$ In $2009 / 2010$, malaria was the leading cause of outpatient visits and health facility admissions, accounting for $14 \%$ of outpatient visits and $9 \%$ of admissions. ${ }^{[4]}$ Chronic malnutrition prevalence reported for the same period, manifesting in the form of stunting and underweight, was $44 \%$ and $29 \%$, respectively, indicating the burden of the problem in the country. ${ }^{[5]}$

The synergy of malnutrition and infections is widely documented elsewhere, ${ }^{[6,7]}$ though the relationship between malnutrition and malaria is conflicting. ${ }^{[8-13]}$ This presents a challenge in attempting to control the issue. This study was conducted to investigate the association between chronic malnutrition and malaria, and provide necessary information to develop effective strategies for the control of malaria and prevention of malnutrition.

\section{Methods \\ Study design and setting}

An institution-based case-control study was conducted to assess the association of undernutrition and malaria among children in BahirDar special zone, which has 10 health centres (3 rural and 7 urban). The zone is situated on the southern shore of Lake Tana, $564 \mathrm{~km}$ north-west of Addis Ababa, with an elevation of $1840 \mathrm{~m}$ above sea level. The zone is heterogeneous with various ethnic populations and malaria is endemic with seasonal transmission, which makes the site ideal for investigating the association of malaria and undernutrition. From the available health facilities at the time of the study, two rural and four urban facilities were purposively selected for our assessment.

\section{Ethical considerations}

Ethical approval was obtained from the School of Public Health Research Ethics Review Committee of Addis Ababa University, College of Health Sciences. Permission was also secured from the Amhara Regional Health Bureau. Officials of each facility were also contacted, and permission was granted to conduct the study. Informed written consent was obtained from each subject for their participation and the right to withdraw from the study at any time was also communicated to all of them. Antimalarial treatment was also provided for children with confirmed malaria and all undernourished children were referred to a nutritional rehabilitation centre, according to the national guideline. 


\section{Sample size determination and subjects}

The required sample size was determined using the double population proportion formula, by considering the magnitude of the two different types of malnutrition of the region: stunting (52.0\%) and underweight $(33.4 \%),{ }^{[4]}$ with level of significance $\alpha=0.05$ and margin of error $d=5 \%$. Power was $80 \%$ with non-response rate of $10 \%$ and expected odds ratio of 1.75 . Two different sample sizes were calculated based on underweight prevalence $(n=621)$ and stunting prevalence $(n=448)$. The sample size that yielded the largest number ( $n=621)$ was taken as our working sample since it accommodated all the above scenarios.

A sample size of 621 was estimated and allocated proportionally across the six health centres: Tis-Abay $(n=108)$, Meshenti $(n=108)$, Shinbit $(n=104)$, Abaymado $(n=100)$, Bahir-Dar $(n=104)$, and Han $(n=97)$. All children who had confirmed malaria were enrolled as cases until the required sample sizes for the respective health centres were reached. For every confirmed malaria case, three controls that visited the facility for any reason other than malaria (upper respiratory tract infection, ear infection, eye infection, minor injury and minor dermal cases) were interviewed based on their order of visiting. Children who had acute lower respiratory tract infection, diarrhoea, measles, severe illness or chronic illness (such as tuberculosis, HIV and AIDS) were excluded from the study.

\section{Data collection and processing}

The important variables included in the questionnaire were sociodemographic, clinical and laboratory data. Twelve data collectors fluent in the local languages of the study zone (two clinical nurses from each health centre), laboratory technicians and two supervisors with relevant experience were recruited and trained for 2 days on the method of data collection. The training addressed issues such as the content of the questionnaire, basic interviewing skills, filling out the questionnaire, and weight and height measurements.

\section{Anthropometric measurements}

Weight was measured in kilograms without shoes, using a digital bath scale with good precision, and recorded to the nearest $100 \mathrm{~g}$. During weight measurement, the scale was repeatedly checked for accuracy against a known weight. Height or length was taken barefoot using a stadiometre. A vertical tape fixed perpendicular to the ground on the wall or recumbent length measurement tape fixed for children under 2 years of age was used and height or length recorded to the nearest centimetre.

\section{Clinical assessments}

Clinical information that included illnesses in the preceding 2 weeks and type of treatments received was assessed by trained outpatient staff of the respective health centre. Oedema was checked by grasping both feet with thumbs on the top of the feet and pressing thumbs gently for 3 seconds (or count 101, 102, 103 if there was no stopwatch); if pitting remained in both feet after lifting the hand, the child was considered as having nutritional oedema. Axillary temperature was also measured using a digital thermometer two times and the average was recorded.

\section{Biological assessments}

A finger prick following aseptic techniques was done on all subjects to prepare thick and thin films for confirmation of malaria parasites, and stained with Giemsa in the respective health centres. Each slide was read by senior laboratory technicians who had participated in the training. Absence of malaria parasite in 200 high-power ocular fields of the thick film was considered as negative.

\section{Statistical analysis}

Data collected in the health centres were checked for completeness, coded, and entered using EpiInfo version 3.5.4 (Centers for Disease Control and Prevention, USA), then exported to SPSS version 21 (IBM,
USA) for cleaning and analysis. For computing the nutritional indices, the data were exported to the WHO's Anthro statistical package version 3.2.2 (Switzerland). Children were classified as stunted (chronic undernutrition) and underweight when the height-for-age $z$-scores (HAZ) and weight-for-age $z$-scores (WAZ) were below minus two $z$-scores, respectively. Severe stunting and underweight were defined when both HAZ and WAZ were below minus three $z$-scores.

The results are presented in percentages and graphs accordingly. Binary logistic regression was employed to examine the associations between sociodemographic variables and undernutrition with malaria and to identify variables for consideration in multivariate analysis. To ascertain the association between the dependent variables and the explanatory variables, simultaneously controlling for the aforementioned explanatory variables (all sociodemographic characteristics and other covariates associated in bivariate with $p<0.2$ were used and entered), stepwise logistic regression was applied and adjusted odds ratios (AORs) and $95 \%$ confidence intervals (CIs) were calculated. Multicollinearity was also checked for WAZ and HAZ using variance inflation factor. In all analyses, $p<0.05$ was considered to be statistically significant.

\section{Results}

Of the total enrolled subjects, 607 (149 cases and 458 controls) participated in the study, making the response rate $96.2 \%$ for cases and $98.3 \%$ for controls. Over three-quarters (78\%) of caregivers were between 20 and 39 years old and most of them were female and married, in both groups. The majority in both groups were housewives and less than half had no formal education. The median household size was five people for both cases and controls, respectively. Less than a quarter were earning above ETB2 000 (USD100) and urban residents constituted more than half in both groups (Table 1).

\section{Measures of children's sociodemographic characteristics and nutritional status}

Various child characteristics are displayed by type of study group (Table 2). As shown, more than half $(58.4 \%)$ of cases and $(51.5 \%)$ of controls were males. Their mean age was 27.5 and 32.2 months for cases and controls, respectively, and this difference was significant $(p=0.04)$. The mean (standard deviation) temperature of cases and controls was $37.8(0.8)^{\circ} \mathrm{C}$ and $36.4(0.5)^{\circ} \mathrm{C}$, respectively. Overall, the proportion of stunting was higher among cases $(50.3 \%)$ than controls $(40.2 \%)$ and the difference was significant $(p=0.01)$. When stunting was further disaggregated by severity, both severe and moderate stunting was higher among cases than controls, though the difference was only significant among those who had severe stunting $(p=0.02)$. Likewise, children who had malaria significantly suffered underweight (34.2\%), more than those without malaria or the control group $(32.3 \%)$. Furthermore, malaria was significantly higher among those suffering moderate underweight $(p=0.01)$.

\section{Identified Plasmodium spp.}

Of the total 149 enrolled malaria cases, 79 (53.0\%) were Plasmodium falciparum, 62 (41.6\%) were P. vivax and the remaining 8 (5.4\%) were mixed cases ( $P$. falciparum and P. vivax; data not shown).

\section{Association of malaria with stunting and underweight}

Overall, stunting was significantly associated with confirmed malaria. The odds of developing malaria were 1.6 times more among stunted than non-stunted children (AOR 1.61, 95\% CI 1.19 - 2.51). Likewise, the odds of developing malaria were 1.7 times more among underweight children than the referent group (AOR 1.69, 95\% CI 1.11 - 2.90; Table 3).

\section{Association of malaria with selected sociodemographic variables}

Place of residence, sex of the child, long-lasting insecticide-treated net (LLIN) utilisation, and use of indoor residual spray (IRS) were 
Table 1. Respondents' characteristics by study groups, BahirDar special zone, Ethiopia, 2014

\begin{tabular}{|c|c|c|c|c|c|c|c|}
\hline Characteristics & $\begin{array}{l}\text { Cases }(N=149), \\
n(\%)\end{array}$ & $\begin{array}{l}\text { Controls }(N=458), \\
n(\%)\end{array}$ & $p$-value & Characteristics & $\begin{array}{l}\text { Cases }(N=149), \\
n(\%)\end{array}$ & $\begin{array}{l}\text { Controls }(N=458), \\
n(\%)\end{array}$ & $p$-value \\
\hline Age (years) & & & & Residence & & & \\
\hline $10-19$ & $3(2.0)$ & $39(8.5)$ & & Urban & $87(58.4)$ & $392(85.6)$ & \\
\hline $20-29$ & $43(28.9)$ & $200(43.7)$ & 0.05 & Rural & $62(41.6)$ & $66(14.4)$ & $0.01^{*}$ \\
\hline $30-39$ & $75(50.3)$ & $163(35.6)$ & 0.90 & Parent of the child & & & \\
\hline $40-49$ & $25(16.8)$ & $52(11.4)$ & $0.01^{*}$ & Alive & $130(87.2)$ & $429(93.7)$ & \\
\hline$\geq 50$ & $3(2.0)$ & $4(0.9)$ & $0.02^{*}$ & Died & $19(12.8)$ & $29(6.3)$ & $0.01^{*}$ \\
\hline Sex & & & & ${ }^{*} p<0.05$. & & & \\
\hline
\end{tabular}

\section{Male}

$20(13.4)$

$74(16.2)$

Female

$129(86.6)$

$384(83.8)$

0.21

Marital status

$\begin{array}{llll}\text { Married } & 129(86.6) & 414(90.4) & \\ \text { Single } & 1(0.7) & 4(0.9) & 0.42 \\ \text { Divorced } & 11(7.4) & 28(6.1) & 0.26 \\ \text { Widowed } & 1(0.7) & 12(2.6) & 0.08 \\ \text { Separated } & 7(4.7) & 0(0.0) & -\end{array}$

Occupation

Housewife $\quad 72(48.3)$

$223(48.7)$

Farmer

$43(28.9)$

$161(35.2)$

0.19

Daily labourer

$18(12.1)$

$23(5.0)$

$45(9.8)$

Employer $\quad 8(5.4)$

Merchant $7(4.7)$

Other

$1(0.7)$

$6(1.3)$

$0(0)$

Educational status

Cannot read and 73 (49.0)

$170(37.1)$

write

Read and write $19(12.8)$

$53(11.6)$

0.27

Primary (Grades 25 (16.8)

$56(12.2)$

0.44

1 - 8)

Secondary

$20(13.4)$

$107(23.4)$

$0.01^{*}$

(Grades 9 - 12)

Grade 12 and

$12(8.0)$

above

Household

members

$\begin{array}{llll}\leq 2 & 5(3.4) & 19(4.1) & \\ 3-4 & 75(50.3) & 158(34.5) & 0.13 \\ \geq 5 & 69(46.3) & 281(61.4) & 0.45 \\ \quad \text { Mean (SD) } & 4.7(1.80) & 5.4(2.15) & \\ \begin{array}{l}\text { Monthly family } \\ \text { income (EB) }\end{array} & & & \\ <500 & 39(26.2) & 79(17.2) & \\ 500-999 & 36(24.2) & 181(39.5) & 0.01^{*} \\ 1000-1999 & 53(35.6) & 133(29.0) & 0.20 \\ \geq 2000 & 21(14.1) & 65(14.2) & 0.09 \\ \text { Mean (SD) } & 1106.3(820.1) & 1026.9(778.5) & \end{array}$

Continued ...
Table 1. (continued) Respondents' characteristics by study groups, Bahir-Dar special zone, Ethiopia, 2014

Table 2. Child characteristics by study groups, Bahir-Dar special zone, June 2014

\begin{tabular}{|c|c|c|c|}
\hline Characteristic & $\begin{array}{l}\text { Cases }(N=149), \\
n(\%)\end{array}$ & $\begin{array}{l}\text { Controls } \\
(N=458), n(\%)\end{array}$ & $p$-value \\
\hline \multicolumn{4}{|l|}{ Sex } \\
\hline Male & $87(58.4)$ & $236(51.5)$ & 0.14 \\
\hline Female & $62(41.6)$ & $222(48.5)$ & \\
\hline \multicolumn{4}{|l|}{$\begin{array}{l}\text { Child age } \\
\text { (months) }\end{array}$} \\
\hline $6-23$ & $70(47.0)$ & $173(37.8)$ & $0.04^{*}$ \\
\hline $24-59$ & $79(53.0)$ & $285(62.2)$ & \\
\hline $\begin{array}{l}\text { Age (months), } \\
\text { mean (SD) }\end{array}$ & $27.5(12.9)$ & $32.2(16.3)$ & $0.01^{*}$ \\
\hline $\begin{array}{l}\text { Temperature } \\
\left({ }^{\circ} \mathrm{C}\right) \text {, mean }(\mathrm{SD})\end{array}$ & $37.8(0.8)$ & $36.4(0.7)$ & $0.01^{\star}$ \\
\hline \multicolumn{4}{|l|}{ Stunting } \\
\hline $\begin{array}{l}\text { Stunted } \\
\text { (overall) }\end{array}$ & $75(50.3)$ & $184(40.2)$ & $0.01^{*}$ \\
\hline Severe stunting & $24(16.1)$ & $63(13.8)$ & $0.02^{*}$ \\
\hline $\begin{array}{l}\text { Moderate } \\
\text { stunting }\end{array}$ & $51(34.2)$ & $121(26.4)$ & 0.10 \\
\hline Normal & $74(49.7)$ & $274(59.8)$ & - \\
\hline \multicolumn{4}{|l|}{ Underweight } \\
\hline $\begin{array}{l}\text { Underweight } \\
\text { (overall) }\end{array}$ & $51(34.2)$ & $102(32.3)$ & $0.01^{*}$ \\
\hline $\begin{array}{l}\text { Severe } \\
\text { underweight }\end{array}$ & $14(9.4)$ & $31(6.8)$ & 0.07 \\
\hline $\begin{array}{l}\text { Moderate } \\
\text { underweight }\end{array}$ & $37(24.8)$ & $71(15.5)$ & $0.01^{*}$ \\
\hline Normal & $98(65.8)$ & $356(77.7)$ & - \\
\hline Total & $149(100)$ & $458(100)$ & \\
\hline${ }^{*} p<0.05$ & & & \\
\hline
\end{tabular}

important variables significantly associated with occurrence of malaria, after controlling for confounding effects in the multivariate model.

Almost one-third (28.9\%) of children in cases and two-thirds (66.2\%) in controls slept the night preceding the interview under a LLIN. As a result, those children who slept under a LLIN were $68.6 \%$ less likely to have confirmed malaria than controls (AOR 0.314, 95\% CI 0.15 - 0.36). Similarly, $42.3 \%$ of children in cases and $73.8 \%$ of controls were from a house sprayed with IRS in the last 12 months. As a result, those children from houses sprayed with IRS were $66.3 \%$ 
Table 3. Correlates of stunting and confirmed malaria in preschool children at Bahir-Dar special zone, 2014, after adjustment for other variables

\begin{tabular}{|c|c|c|c|c|}
\hline Variable & Cases $(N=149), n(\%)$ & Controls $(N=458), n(\%)$ & Crude OR (95\% CI) & AOR (95\% CI) \\
\hline \multicolumn{5}{|l|}{ Stunting } \\
\hline Yes & $75(50.3)$ & $184(40.2)$ & $1.509(1.041-2.188)$ & \multirow[t]{2}{*}{$1.614\left(1.192-2.514^{*}\right)$} \\
\hline No & $74(49.7)$ & $274(59.8)$ & 1 & \\
\hline \multicolumn{5}{|l|}{ Underweight } \\
\hline Yes & $51(34.2)$ & $102(22.3)$ & $1.816(1.213-2.720)$ & \multirow[t]{2}{*}{$1.690\left(1.112-2.903^{\star}\right)$} \\
\hline No & $98(65.8)$ & $356(77.7)$ & 1 & \\
\hline \multicolumn{5}{|l|}{ Residence } \\
\hline Rural & $62(41.6)$ & $66(14.4)$ & $4.233(2.789-6.424)$ & $1.546\left(1.007-3.179^{\star}\right)$ \\
\hline Urban & $87(58.4)$ & $392(85.6)$ & 1 & 1 \\
\hline \multicolumn{5}{|l|}{ Sex } \\
\hline Male & $87(58.4)$ & $236(51.5)$ & $1.320(0.908-1.918)$ & $1.416(0.931-1.980)$ \\
\hline Female & $62(41.6)$ & $222(48.5)$ & 1 & 1 \\
\hline \multicolumn{5}{|l|}{ Child age (months) } \\
\hline $6-23$ & $70(47.0)$ & $173(37.8)$ & $0.685(0.472-0.995)$ & $1.128(0.649-1.676)$ \\
\hline $24-59$ & $79(53.0)$ & $285(62.2)$ & 1 & 1 \\
\hline \multicolumn{5}{|l|}{ Slept under LLIN last night } \\
\hline Yes & $43(28.9)$ & $303(66.2)$ & $0.208(0.139-0.311)$ & $0.314\left(0.148-0.360^{\star}\right)$ \\
\hline No & $106(71.1)$ & $155(33.8)$ & 1 & 1 \\
\hline \multicolumn{5}{|c|}{ Indoor residual spray within last 12 months } \\
\hline Yes & $63(42.3)$ & $338(73.8)$ & $0.260(0.177-0.383)$ & $0.337\left(0.204-0.530^{\star}\right)$ \\
\hline No & $86(57.7)$ & $120(26.2)$ & 1 & 1 \\
\hline \multicolumn{5}{|l|}{ Education status } \\
\hline Cannot read and write & $73(49.0)$ & $170(37.1)$ & 1 & 1 \\
\hline Read and write & $19(12.8)$ & $53(11.6)$ & $0.835(0.462-1.508)$ & $0.811(0.401-1.716)$ \\
\hline Grades $1-12$ & $45(30.2)$ & $163(35.6)$ & $0.643(0.419-0.987)$ & $0.778(0.510-1.311)$ \\
\hline Above Grade 12 & $12(8.1)$ & $72(15.7)$ & $0.388(0.199-0.758)$ & $0.618(0.414-1.286)$ \\
\hline \multicolumn{5}{|l|}{ Occupation } \\
\hline Housewife & $72(48.3)$ & $223(48.7)$ & 1 & 1 \\
\hline Farmer & $43(28.9)$ & $161(35.2)$ & $0.827(0.539-1.270)$ & $0.880(0.487-1.884)$ \\
\hline Merchant or other & $26(17.4)$ & $29(6.3)$ & $2.777(1.536-5.021)$ & $1.621(0.988-7.164)$ \\
\hline Employer & $8(5.4)$ & $45(9.8)$ & $0.551(0.248-1.222)$ & $0.994(0.553-2.046)$ \\
\hline${ }^{*} p<0.05$ & & & & \\
\hline
\end{tabular}

less likely (AOR 0.33, 95\% CI 0.20 - 0.53) to have confirmed malaria than those who did not, after controlling for nutritional and other sociodemographic variables.

Males were 1.4 times more likely to have confirmed malaria than females, though the difference was not significant (AOR 1.41, 95\% CI 0.93 - 1.98). Children from rural areas were 1.5 times more likely to have confirmed malaria than those from urban areas after stunting, underweight and other important factors were controlled (AOR 1.5, 95\% CI 1.01 - 3.18).

\section{Discussion}

This facility-based case-control study attempted to establish the association between chronic undernutrition and malaria, as well as other important sociodemographic variables of major public health significance in this and other developing countries, to enable the incorporation of programme initiatives in malaria prevention and control guidelines.

In this study, chronic undernutrition, in the form of stunting and underweight, was common, and undernourished children were at higher risk of contracting malaria than well-nourished children. The odds of having confirmed malaria were 1.6 times higher among stunted children than non-stunted children and this finding was consistent with a longitudinal follow-up study conducted in Gambia and a cross-sectional study in Kenya, both of which reported that stunted children were at higher risk of developing malaria. ${ }^{[8,9]}$ Furthermore, Fillol et al. ${ }^{[10]}$ through a cellular-level nested case-control study, substantiated the aforementioned findings by showing that antimalarial immune response is significantly lower among stunted children, suggesting that undernutrition could predispose to clinically overt malaria. Our study enrolled symptomatic malaria cases that could have been a result of lower antimalarial immune response, even though the study did not differentiate whether cases were new malaria infections or subsequent malarial attacks. In contrast to these findings, a Senegalese cohort study of young children from rural areas, a Ghanaian study and western Ethiopian studies have all reported no association between stunting and malaria infection. ${ }^{[1-13]}$ The discrepancy could be explained by the difference in case definition used in both studies - the studies in Ghana and Ethiopia compared asymptomatic malaria 
and the Senegal study focused on patients with chronic malarial attack, differing entirely from the present study which used confirmed malaria cases. In addition, the Ghanaian study had sample size limitations, unlike the present study which had a large sample size and controlled for the confounding effects of important variables. The study in Ethiopia also found low malaria prevalence, making the analysis non-significant. ${ }^{[13]}$ The comparative nature of our study alleviated the problem of low malaria prevalence, enabling comparison of the relationships with adequate sample size for cases and controls.

Similarly, the odds of having a confirmed malaria case were 1.7 times higher among underweight children than their counterparts and this finding is concordant with a WHO Comparative Risk Assessment project, which found that children who were moderately to severely underweight had an increased risk of a clinical malaria attack, compared with those who were better nourished, although this difference was not significant. ${ }^{[14]}$ Nonsignificance could have been due to low prevalence of clinical malaria, which was described as a study limitation. A similar explanation was put forward by a northern Guinea study, which used a large sample size of 4000 children. ${ }^{[15]}$ The higher odds of confirmed malaria among underweight children are because of increased susceptibility to malaria for different reasons, such as through a reduction in the function of the immune system. A study conducted by Scrimshaw and SanGiovanni ${ }^{[16]}$ indicated that undernourished children are unable to mount an appropriate immune response to the malaria parasite due to a reduction in T-lymphocytes, impairment of antibody formation, decreased complement formation, and atrophy of thymus and other lymphoid tissues. In contrast to the present findings, a study in Port Harcourt, Nigeria, showed that underweight children were not at a higher risk of asymptomatic malaria attack than wellnourished children (RR 1.02, 95\% CI $0.34-2.37$ ). ${ }^{[17]}$ The discrepancy could be due to the difference in cases, as asymptomatic malaria cases were used, unlike our study, which enrolled confirmed symptomatic cases. Similarly, studies in Kenya and Uganda had reported no relationship between baseline nutritional status and subsequent incidence of malaria. ${ }^{[18,19]}$ The discrepancy noted with the present study could be explained by malaria transmission in Uganda being holoendemic and perennial, while in the present study transmission is seasonal. The Kenyan study included children up to 8 years old, ${ }^{[18]}$ another difference from our study.

Other important factors strongly associated with malaria attack were use of LLIN for children during sleeping, use of IRS in the last 12 months and place of residence.

Use of LLIN for children during sleeping was strongly associated with clinical malaria after controlling for undernutrition and other demographic variables. Those children who slept under LLIN were $68.6 \%$ less likely to have confirmed malaria than children who did not. This is concordant with a community-based comparative interventional study conducted in Nigeria, which showed a $93 \%$ decrease in malaria prevalence for those who slept under LLIN compared with those who did not. ${ }^{[20]}$

Children from houses sprayed with IRS in the last 12 months were $66.3 \%$ less likely to have confirmed malaria than their counterparts, after controlling for undernutrition and other confounders.

In this study, children from rural areas were 2.3 times more likely to have confirmed malaria than children from urban areas. This could be explained by higher irrigation and drainage in rural than urban areas, which is favourable for mosquito breeding and malaria transmission.

The strengths of the study are that it compared cases and controls using an appropriate sample size, included anthropometric measurements, and used the laboratory for confirmation of malaria diagnosis, to decrease the subjectivity of diagnosis/bias. Micronutrient status, anaemia and the level of parasitaemia, which might have an impact, were not assessed and are considered limitations of the study.

\section{Conclusion}

Chronic undernutrition was closely associated with malaria infection. The major predictors for contracting malaria were being stunted, underweight, rural residence, not using LLIN or IRS, male sex, and low educational status of the mother/caregiver. It is recommended that health offices and non-government organisations operating in malaria endemic areas distribute LLINs for households and create awareness on the importance of maintaining good nutrition, as well as implementing other preventive measures addressing the previously mentioned predictors. Integration of malaria intervention programmes with essential nutrition actions would be an effective strategy to reduce morbidity and mortality from malaria and undernutrition. Further studies with more biochemical tests might be helpful in addressing some of the study limitations.

Acknowledgement. The Addis Ababa School of Public Health Research Ethics Review Committee, and the staff from Bahir-Dar health offices, the respective health centres and the municipality, are duly acknowledged. This work would not have reached its present state without the unreserved participation of the respondents and the support rendered by our families.

\section{References}

1. World Health Organization (WHO). Global Malaria Programme: World Malaria Report 2012. www.who.int/malaria (accessed 10 December 2013).

2. Food and Agricultural Organization of the United Nations (FAO). The State of Food Insecurity in the World, 2012. http://www.fao.org/docrep/016/i3027e/ i3027e00.htm (accessed 13 February 2014).

3. United Nations International Children's Fund (UNICEF). Improving child nutrition: The achievable imperatives for global progress. New York: UNICEF, 2013.

4. Ethiopian Health and Nutrition Research Institute (EHNRI). Ethiopian malaria indicator survey report 2011. Addis Ababa, Ethiopia: EHNRI, 2011.

5. Central Statistical Agency (Ethiopia) and ICF International. 2012. Ethiopia Demographic and Health Survey 2011. Addis Ababa, Ethiopia and Calverton, USA: Central Statistical Agency and ICF International, 2011:156-157.

6. Cunningham RS, McNeeley DF, Moon A. Mechanisms of nutrient modulation of the immune response. J Allergy Clin Immunol 2005;115(1129):1119-1128. [http://dx.doi.org/10.1016/j.jaci.2005.04.036]

7. Roth DE, Caulfield LE, Ezzati M, Black RE. Acute lower respiratory infections in childhood: Opportunities for reducing the global burden through nutritional interventions. Bull World Health Organ 2008;86(4):356-364. [http://dx.doi. org/10.2471/blt.07.049114]

8. Deen JL, Walraven GE, von Seidlein L. Increased risk for malaria in chronically malnourished children under 5 years of age in rural Gambia. J Trop Pediatr 2002;48(2):78-83. [http://dx.doi.org/10.1093/tropej/48.2.78]

9. Friedman JF, Kwena AM, Mirel LB, et al. Malaria and nutritional status among pre-school children: Results from cross-sectional surveys in Western Kenya. Am J Trop Med Hyg 2005;73(4):698-704.

10. Fillol F, Sarr JB, Boulanger D, et al. Impact of child malnutrition on the specific anti-Plasmodium falciparum antibody response. Malar J 2009;8(2):116. [http:// dx.doi.org/10.1186/1475-2875-8-116]

11. Fillol F, Cournil A, Boulanger D, et al. Influence of wasting and stunting at the onset of the rainy season on subsequent malaria morbidity among rural preschool children in Senegal. Am J Trop Med Hyg 2009;80(2):202-208.

12. Crookston BT, Alder SC, Boakye I, et al. Exploring the relationship between chronic undernutrition and asymptomatic malaria in Ghanaian children. Malar J 2010;9(2):39. [http://dx.doi.org/10.1186/1475-2875-9-39]

13. Deribew A, Alemseged F, Tessema F, et al. Malaria and undernutrition: A community based study among under-five children at risk of malaria, south-west Ethiopia. PLoS One 2010;5(5):e10775. [http://dx.doi.org/10.1371/journal.pone.0010775]

14. Caulfield LE, Richard SA, Black RE. Under-nutrition as an underlying cause of malaria morbidity and mortality in children less than five years old. Am J Trop Med Hyg 2004;71(2 Suppl):55-63.

15. Snow RW, Byass P, Shenton FC, Greenwood BM. The relationship between anthropometric measurements and measurements of iron status and susceptibility to malaria in Gambian children. Trans R Soc Trop Med Hyg 1991;85(5):584-589.

16. Scrimshaw NS, SanGiovanni JP. Synergism of nutrition, infection, and immunity: An overview. Am J Clin Nutr 1997;66(2):464S-477S.

17. Jeremiah ZA, Uko EK. Childhood asymptomatic malaria and nutritional status among Port Harcourt children. East Afr J Public Health 2007;4(2):55-58.

18. Nyakeriga AM, Troye-Blomberg M, Chemtai AK, Marsh K, Williams TM. Malaria and nutritional status in children living on the coast of Kenya. Am J Clin Nutr 2005;80(23):1604-1610. [http://dx.doi.org/10.1111/j.03009475.2004.01423o.x]

19. Arinaitwe E, Gasasira A, Verret W, et al. The association between malnutrition and the incidence of malaria among young HIV-infected and -uninfected Ugandan children: A prospective study. Malar J 2012;27(11):90. [http://dx.doi. org/10.1186/1475-2875-11-90]

20. Ashikeni MA, Envuladu EA, Zoakah AI. Malaria and the use of the insecticidetreated net (ITN) among under-five children in Kuje Area Council of the Federal Capital Territory Abuja, Nigeria. J Mosq Res 2013;3(6):45-53. [http:// dx.doi.org/10.5376/jmr.2013.03.0006]. 